\title{
A Case Study of Holomorphic Semigroups
}

\author{
Bishnu Hari Subedi ${ }^{1}$, Ajaya Singh ${ }^{2}$ \\ 1,2 Central Department of Mathematics, Institute of Science and Technology, \\ Tribhuvan University, Kirtipur, Kathmandu, Nepal \\ Correspondence to: Bishnu Hari Subedi, Email: subedi.abs@gmail.com
}

\begin{abstract}
In this paper, we investigate some characteristic features of holomorphic semigroups. In particular, we investigate nice examples of holomorphic semigroups whose every left or right ideal includes minimal ideal. These examples are compact topological holomorphic semigroups.
\end{abstract}

Keywords: Holomorphic semigroup, Ideals, Abundant semigroup, Compact topological semigroup DOI: https://doi.org/10.3126/jnms.v3i2.33958

\section{Introduction}

Semigroups are very classical algebraic structures with a binary composition that satisfies associative law. Semigroups naturally arose from the general mapping of a set into itself. Therefore, a set of holomorphic functions on the complex plane $\mathbb{C}$ or Riemann sphere $\mathbb{C}_{\infty}$ or certain subsets thereof naturally forms a semigroup. We call that any analytic map on $\mathbb{C}$ or $\mathbb{C}_{\infty}$ or on certain subsets thereof by a holomorphic function.

Definition 1.1 (Holomorphic semigroup and subsemigroup). $A$ holomorphic semigroup $S$ is a semigroup of holomorphic functions defined on $\mathbb{C}$ or $\mathbb{C}_{\infty}$ or certain subsets thereof with the semigroup operation being functional composition. A non-empty subset $T \subseteq S$ is a subsemigroup of $S$ if $f \circ g \in T$ for all $f, g \in S$.

Let

$$
\mathscr{F}=\left\{f_{\alpha}: f_{\alpha} \text { is a holomorphic function for all } \alpha \in \Delta\right\},
$$

where index set $\Delta$ is allowed to be infinite in general. We are interested in special holomorphic semigroups whose each element can be expressed as a finite composition of certain holomorphic functions. More formally, such a semigroup is defined as follows:

Definition 1.2 (Holomorphic semigroup generated by holomorphic functions). Let $\mathscr{F}$ be a family of holomorphic functions as defined in (1.1). A holomorphic semigroup $S$ generated by $\mathscr{F}$ is a semigroup of all elements that can be expressed as a finite composition of elements in $\mathscr{F}$. We denote such holomorphic semigroup by $S=\left\langle f_{\alpha}\right\rangle_{\alpha \in \Delta}$ or simply $S=\left\langle f_{\alpha}\right\rangle$.

Holomorphic semigroup $S$ is said to be a rational semigroup or a transcendental semigroup depending on whether $\mathscr{F}$ is a collection of rational functions or transcendental entire functions. In particular, $S$ is said to be a polynomial semigroup if $\mathscr{F}$ is a collection of polynomials of degree at least one. The transcendental semigroups and polynomial semigroups are also called entire semigroups. Dynamical properties of rational and transcendental semigroups were studied first by Hinkannen and Martin [2] and Poon 3 respectively. In the papers [4, 5, 6], we investigated further dynamical features of holomorphic semigroups. In this paper, we deal only algebraic aspect of holomorphic semigroups.

A holomorphic semigroup $S$ is abelian if $f_{\alpha} \circ f_{\beta}=f_{\beta} \circ f_{\alpha}$ for all generators $f_{\alpha}, f_{\beta}$ of $S$. By definition, each $f \in S$ is constructed through the composition of finite number of functions $f_{\alpha}$ for $\alpha \in \Delta$. That is, $f=f_{\alpha_{1}} \circ f_{\alpha_{2}} \circ \cdots \circ f_{\alpha_{m}}$ for some $m \in \mathbb{N}$ and $\alpha_{1}, \alpha_{2}, \ldots \alpha_{m} \in\{1,2, \ldots, m\}$. A semigroup generated by finitely many holomorphic functions $f_{j},(j=1,2, \ldots, n)$ is called a finitely generated holomorphic semigroup, and we write $S=\left\langle f_{1}, f_{2}, \ldots, f_{n}\right\rangle$. If $S$ is generated by only one holomorphic function $f$, then $S$ is called a cyclic holomorphic semigroup, and we write $S=\langle f\rangle$. In this case, each $g \in S$ can be written as $g=f^{n}$, where $f^{n}$ is the $n$th iterate of $f$ with itself.

By the definition of a holomorphic semigroup, we at once get the following assertion. 
A Case Study of Holomorphic Semigroups

Theorem 1.1 ([5, Proposition 1.1]). Let $S=\left\langle f_{\alpha}\right\rangle$ be a holomorphic semigroup. Then for every $f \in S, f^{m}$ (for all $m \in \mathbb{N}$ ) can be written as $f^{m}=f_{\alpha_{1}} \circ f_{\alpha_{2}} \circ f_{\alpha_{3}} \circ \cdots \circ f_{\alpha_{p}}$, where $\alpha_{i} \in\{\alpha: \alpha \in \Delta\}$, for some $p \in \mathbb{N}$.

Example 1.1. Let $S$ be a set consisting all of powers $z$ which are either all powers of 2 or all powers of 3 or product of all powers of 2 and 3. Then $S$ forms a semigroup under functional composition. It is a finitely generated polynomial semigroup generated by two polynomials $z \rightarrow z^{2}$ and $z \rightarrow z^{3}$. In this case, $S=\left\langle z^{2}, z^{3}\right\rangle$.

Example 1.2. Let $\alpha \in \mathbb{C}$ such that $\Re \alpha \geq 0$. For any $k \in \mathbb{N}$, the function $f_{k}(z)=e^{-\alpha k} z$ for all $z \in \mathbb{C}$ is holomorphic in the complex plane $\mathbb{C}$, and so $S=\left\{f_{k}: k \in \mathbb{N}\right\}$ is a holomorphic (in particular, entire) semigroup. Also, each $f \in S$ can be written as $f(z)=f_{1}^{l}(z)$ for some $l \in \mathbb{N}$. Therefore, $S=\left\langle f_{1}\right\rangle$.

Example 1.3. If we choose $\Delta$ a set of positive rational numbers $\mathbb{Q}^{+}$in Example 1.2, then each function $f_{r}(z)=e^{-\alpha r} z$ for all $z \in \mathbb{C}$ and $r \in \mathbb{Q}^{+}$for some $\alpha \in \mathbb{C}$ such that $\Re \alpha \geq 0$, can be written as a finite composition of $f_{t}(z)=e^{-\alpha t} z$ with $t \in \mathbb{Q}^{+}$. Hence $S=\left\langle f_{t}\right\rangle_{t \in \mathbb{Q}^{+}}$is a holomorphic semigroup generated by the set $\left\{f_{t}: t \in \mathbb{Q}^{+}\right\}$.

Note that these are examples of abelian holomorphic semigroups. The following holomorphic semigroups are non-abelian.

Example 1.4. $S_{1}=\left\langle e^{z}, e^{-z}\right\rangle, S_{2}=\langle\sin z, \cos z\rangle$.

\section{Ideal Theory of Holomorphic Semigroups}

There are certain subsets of semigroups with a stronger closure property rather than that of subsemigroups.

Definition 2.1 (Left ideal, right ideal and two sided ideal). Let I be a non-empty subset of holomorphic semigroup $S$. We say $I$ is a left ideal (or right ideal) of $S$ if $f \circ h \in I$ (or $h \circ f \in I$ ) for all $f \in S$ and $h \in I$, that is, $S I \subset I$ (or $I S \subset I$ ). We say $I$ is two sided ideal (or simply ideal) if it is both left and right ideal.

Note that if $S$ is an abelian semigroup, then the notions of left ideal, right ideal, and two sided ideal coincide. For any non-empty subset $K$ of a holomorphic semigroup $S$, the sets

$$
\begin{aligned}
& S K=\{f \circ g: f \in S, g \in K\}=\bigcup_{g \in K} S \circ g, \\
& K S=\{g \circ f: f \in S, g \in K\}=\bigcup_{g \in K} g \circ S,
\end{aligned}
$$

and

$$
S K S=\{f \circ g \circ h: f, h \in S, g \in K\}=\bigcup_{g \in K} S \circ g \circ S
$$

are respectively left, right and two sided ideals. Likewise, for any $g \in S$, the sets $S \circ g, g \circ S$ and $S \circ g \circ S$ are respectively left, right and two sided ideals. In general, $g$ may not be in $g \circ S$ ( or $S \circ g$ or $S \circ g \circ S$ ) for each $g \in S$. If it happens to be in $g \circ S$ ( or $S \circ g$ or $S \circ g \circ S$ ), then $g=g \circ f$ ( or $g=f \circ g$ or $g=f \circ g \circ h$, $h \in S$ ) for some $f \in S$. In this case, $g \circ S$ (or $S \circ g$ or $S \circ g \circ S$ ) is a smallest right (or left or two sided) ideal containing $g$, which is a right (or left or two sided) ideal generated by $g$. Otherwise, $g \circ S$ (or $g \circ S$ or $S \circ g \circ S$ ) is said to be quasi-generated by $g$. It is obvious that the union of any non-empty family of left (or right or two sided) ideals of $S$ is a left (or right or two sided) ideal of $S$.

On the basis of some topological structure of the complement of $g \circ S$ in $S$, we can define the following types of holomorphic semigroups. Recall that a space $X$ is compact if every open cover of $X$ contains a finite subcover.

Definition 2.2 (F-semigroup and C-semigroup). Let $S$ is a holomorphic semigroup and $g \in S$. Then we say

1. $S$ is a F-(right) semigroup if $S-g \circ S$ is finite; 
Journal of Nepal Mathematical Society (JNMS), Vol.3, Issue 2 (2020); B. H. Subedi and A. Singh

2. $S$ is a $C$-(right) semigroup if $S-g \circ S$ is relatively compact (that is, $\overline{S-g \circ S}$ is compact in $S$ ).

Analogously, we can define $F$-(left) semigroup and $C$-(left) semigroup of any holomorphic semigroup $S$. We say only $F$-semigroup and $C$-semigroup onward for such holomorphic semigroup $S$ on the assumption that left/right is clear from the context. For example, holomorphic semigroup of Example 1.2 is both $F$-semigroup and C-semigroup and that of Examples 1.1 and 1.3 are $C$-semigroups.

There are certain type of left (or right) ideals which are connected to two sided ideals. That is, on the basis of such ideals of holomorphic semigroups, we can construct two sided ideals. This ideal structure is defined as follows.

Definition 2.3 (Minimal left (or right) ideal). A left (or right) ideal $M$ of holomorphic semigroup $S$ is minimal if for every left (or right) ideal $I$ of $S$ such that $I \subseteq M$, then we have $M=I$.

Note that minimal left (or right) ideal of $S$ may be empty. If it is non-empty for a certain holomorphic semigroup $S$, then for every $f \in M$, there must be $M \circ f=M$ (or $f \circ M=M$ ) and $S \circ f=M$ (or $f \circ S=M)$. Also, a minimal left (or right) ideal is always contained in every two sided ideal of $S$. We can also make two sided ideal by the help of minimal left (or right) ideals. For any holomorphic semigroup $S$, let us define

$$
K(S)=\bigcup\{M: M \text { is a minimal left (or right) ideal of } S\}
$$

Since $K(S)$ is non-empty if and only if $S$ has at least one minimal left (or right) ideal and in such a case it is itself a minimal left (or right) ideal. So, as stated above, it is contained in every two sided ideal of $S$. Since for all $f \in S$

$$
K(S) \circ f=\bigcup\{M \circ f: M \text { is a minimal left (or right) ideal of } S\} \subseteq K(S)
$$

So, $K(S)$ is also left (or right) ideal as well. From this discussion, we can conclude the following result.

Proposition 2.1 (Example of minimal two sided ideal). For any holomorphic semigroup $S, K(S)$ is a minimal two sided ideal of $S$ if it is non-empty.

Proof. See for instance in [1, Theorem 2.9].

We can define a special type of holomorphic semigroup $S$ where $K(S)$ is non-empty. Such a semigroup has some special features such as every left (or right) ideal of $S$ include minimal one and every left (or right) ideal of $S$ contains a special element which is called an idempotent. Recall that an element $e \in S$ is an idempotent if $e \circ e=e$.

Definition 2.4 (Abundant semigroup). A holomorphic semigroup $S$ is abundant if every left (or right) ideal of $S$ includes a minimal one and every minimal left (or right) ideal contains an idempotent element.

It is obvious that $K(S) \neq \emptyset$ if $S$ is abundant. There is a topologically significant nice examples of abundant semigroups which can be defined as follows.

Definition 2.5 (Compact right topological holomorphic semigroup). Let $S$ be a holomorphic semigroup and $g \in S$.

1. We define a right translation map $F_{g}: S \rightarrow S$ with respect to $g$ by $F_{g}(h)=h \circ g$ for all $h \in S$.

2. We define a compact holomorphic right topological semigroup by the pair $(S, \tau)$, where $\tau$ is a topology on $S$ such that the space $(S, \tau)$ is compact and Hausdorff and right translation map $F_{g}$ for every $g \in S$ is continuous with respect to $\tau$.

Note that a left translation map and a compact holomorphic left topological semigroup are defined similarly. Also note that in a compact right topological semigroup, we do not require that left translation maps are continuous. We say only translation map and compact holomorphic topological semigroup if left or right is clear from the context. 
Example 2.1. Let $S=\left\langle f_{t}: t \in \mathbb{Q}\right\rangle$ be a holomorphic semigroup, where $f_{t}$ is a function of Example 1.3 for all $t \in \mathbb{Q}$. The collection of all subsets of $S$ forms a topology $\tau$ on $S$ and hence it is also open cover of $S$. There are some finite number of elements in $\tau$ that can cover $S$. Hence, the space $(X, \tau)$ is compact and Hausdorff as well. Therefore, this semigroup is compact holomorphic topological semigroup and hence abundant.

Note that in the holomorphic semigroup $S$ of Example 2.1. there is an element $f_{0}(z)=e^{-\alpha 0} z$ such that $f_{0} \circ f_{0}=f_{0}$ which an idempotent by definition. Therefore, unless holomorphic semigroup in general, abundant semigroup and in particular, compact holomorphic topological semigroup do have idempotents.

Theorem 2.1 (Idempotents exist for holomorphic semigroup). Let $S$ be a compact holomorphic topological semigroup. Then there is an element $e \in S$ such that $e \circ e=e$.

This theorem can be proved as a standard application of Zorn's lemma from set theory. It states that if every chain $\mathscr{C}$ in a partially ordered set $(S, \leq)$ has upper bound in $S$, then $(S, \leq)$ has a maximal element. Note that partial ordered set is a system consisting of non-empty set $S$ and a relation denoted by $\leq$ satisfying the properties of anti-symmetry, reflexivity and transitivity. A chain $\mathscr{C}$ in a partial ordered set $(S, \leq)$ is subset of $S$ such that for every $x, y \in \mathscr{C}$, either $x \leq y$ or $x \geq y$. An element $m \in S$ is a maximal element of $(S, \leq)$ if $m \leq x$ for $x \in S$ implies $m=x$.

Sketch of the Proof of Theorem 2.1. The proof of this theorem follows via the following two facts:

Fact 1: $S$ has a minimal close subsemigroup.

Let $\tau$ be a family of all closed subsemigroups of semigroup $S$. Then $\tau \neq \emptyset$ and it is a topology of closed sets partially ordered by the reverse inclusion. Let $\mathscr{C} \subset \tau$ be a chain in $(\tau, \supseteq)$. Since $S$ is compact and $\mathscr{C}$ has the finite intersection property. So, $\cap_{T \in \mathscr{C}} T$ is non-empty and serves as a least upper bound of $\mathscr{C}$. Then by Zorn's lemma, $\tau$ has a maximal element $M$ (say) where $M \subseteq \cap_{T \in \mathscr{C}} T$. In reality of this context, $M$ is minimal closed subsemigroup of $S$.

Fact 2: If $e \in M$, then $M=\{e\}$ and $e$ is an idempotent.

We can consider two cases of the proof of this fact 2 .

Case 1: We prove $M=M \circ e=\{e\}$. Let $e \in M$. Then $M \circ e$ is a subsemigroup of $M$. The map $F_{e}: M \rightarrow M \circ e, F_{e}(h) \rightarrow h \circ e$ is a right translation map of topological holomorphic semigroup $S$ restricted to $M$. Then it is continuous. Since $M$ is compact, so $M \circ e$ is also compact as a image of compact set under continuous map $F_{e}$. Since by fact $1, M$ is minimal and $M \circ e$ is non-empty, we must $M=M \circ e=\{e\}$. This proves that $e$ is an idempotent.

Case 2: We prove $N=\{f \in M: f \circ e=e\}=M$. By the construction of set $N$, it is subset of $M$ and closed under functional composition. This shows that $N$ is a subsemigroup of $M$. By the case $1, e \in M=M \circ e$ can be written as $e=f \circ e$ for some $f \in M$. This shows that $e \in N \neq \emptyset$. Finally, $N$ can be written as the intersection of closed subsets of $M$ and $F_{e}^{-1}\{\{e\}\}$. This proved $N$ is a non-empty closed subsemigroup of $M$. Then as in the case $1, N=\{e\}$.

Since every left (or right) ideal is a subsemigroup of $S$. So, from the proof of Theorem 2.1, we can say the following important facts for every compact holomorphic topological semigroup $S$.

1. Every left (or right) ideal of the form $S \circ f($ or $f \circ S$ ) is the image of continuous right translation map $F_{f}(g)=g \circ f$ for all $g \in S$. So $S \circ f($ or $f \circ S$ ) is closed in $S$.

2. For every left (or right) ideal $I$ of $S$, we have $S \circ f \subset I$ (or $f \circ S \subset I$ ). Thus every left (or right) ideal contains closed ideal.

3. For every minimal left (or right) ideal $M$ of $S$, we have $M=S \circ f$ (or $M=f \circ S$ for all $f \in M$. This shows that every minimal left (or right) ideal of $S$ is closed.

4. Every left (or right) ideal contains an idempotent element.

5. Let $I \subset J$, where $J$ is an arbitrary left (or right) ideal, and $I$ is a closed left (or right) ideal of $S$. By Zorn's lemma, there is a minimal element $L$ of $(\mathcal{I}, \subseteq$ ), where $\mathcal{I}$ is the family of closed left (or right) ideals contained in $I$. This shows $L$ is a minimal left (or right) ideal of $S$. That is, every left (or right) ideal $S$ contains minimal left (or right) ideal. 
From all of above facts, we may conclude the following assertion.

Theorem 2.2 (An example of abundant semigroup). Every compact holomorphic topological semigroup is abundant.

There is a close connection between minimal left ideals and certain idempotents (minimal idempotents). If we suppose a set of idempotents of arbitrary holomorphic semigroup by $E(S)$, then it may be empty but it is non-empty for abundant holomorphic semigroup. Minimal idempotent can be defined in a partial order relation on $E(S)$.

Definition 2.6 (Dominated element of set of idempotents). Let $E(S)$ be a set of idempotents of (abundant) holomorphic semigroup $S$. We say e is domonated by $f$ on $E(S)$ and write

$$
e \leq f \text { if and only if } e \circ f=f \circ e=e \text { for all } e, f \in E(S) \text {. }
$$

This is clearly partial order relation on $E(S)$. That is, for all $e, f, g \in E(S)$, we have

1. $e \leq e$.

2. $e \leq f$ and $f \leq e \Longrightarrow e=f$.

3. $e \leq f$ and $f \leq g \Longrightarrow e \leq g$.

An element $e \in E(S)$ is minimal if there is no element of $E(S)$ strictly less than $e$. That is, if $h \leq e$, then $h=e$ for all $h \in E(S)$. In a abundant holomorphic semigroup, minimal idempotents are tightly connected to minimal left (or right) ideals.

Theorem 2.3 (A connection between minimal ideal and minimal idempotant). Let $S$ be a holomorphic abundant semigroup and $e \in E(S)$.

1. If $L \subseteq S \circ e$ (or $L \subseteq e \circ S$ ) is a minimal left (or right) ideal, then there is some idempotent $f \in L$ such that $f \leq e$.

2. $e$ is a minimal idempotent if and only if $e \in L$ for some minimal left (or right) ideal $L$, that is, if and only if $e \in K(S)$.

3. $e$ is minimal idempotent if and only if the left (or right) ideal $L=S \circ e($ or $L=e \circ S$ ) is minimal.

4. There is some minimal idempotent $f$ such that $f \leq e$.

Proof. We prove this theorem for left ideal $L$ of semigroup $S$. By symmetry, the same is true for right ideal.

(1) Since $L$ is minimal left (or right) ideal, so there an idempotent in $L$. Since $S$ is abundant, so there is an $i \in L \cap E(S)$. Since $i \in L \subseteq S \circ e$, so $i=s \circ e$ for some $s \in S$. Now

$$
i \circ e=s \circ e \circ e=s \circ e=i \text {. }
$$

Let $e \circ i=f$. We will show that $f$ is our required element. First of all $f \in L$ because $L$ is a left ideal. Secondly, $f$ is an idempotent because

$$
f \circ f=e \circ i \circ e \circ i=e \circ i \circ i=e \circ i=f .
$$

Finally, $f$ is an minimal idempotent because

$$
e \circ f=e \circ e \circ i=e \circ i=f
$$

and

$$
f \circ e=e \circ i \circ e=e \circ i=f .
$$

(2) Let us suppose that $e$ is a minimal idempotent of $S$. Let us make a set

$$
\mathcal{L}=\{L \subseteq S: L \text { is a minimal left ideal of } S\}
$$


Since $S$ is abundant, so there is $L \in \mathcal{L}$ such that $L \subseteq S \circ e$. By (1), there is $f \in L \cap E(S)$ such that $f \leq e$. Since $e$ is a minimal idempotent, we must have $f=e$. Therefore, $e \in L$. Conversely assume that $e \in L$ for some minimal left ideal $L \in \mathcal{L}$. We have to show that $e$ is an minimal idempotent. Let $h \in E(S)$ with $h \leq e$. Then $S \circ h \subseteq S \circ e$. By minimality of $L$, we can write

$$
L=S \circ h=S \circ e .
$$

So $e=g \circ h$ for some $g \in S$. By $h \leq e$, we can write $h=e \circ h$ and so

$$
h=e \circ h=g \circ h \circ h=g \circ h=e .
$$

This proves $e$ is a minimal idempotent.

(3) This follows from the observation of converse part of (b) that if $e \in L$ and $L$ is minimal left ideal, then $L=S \circ e$.

(4) By abundancy of $S$ and (1), there is a minimal left ideal such that $L \subseteq S \circ e$ and $f \in L \cap E(S)$ such that $f \leq e$. Then $f$ is minimal by $(2)$.

From the proof of Theorem 2.3, it is not hard to conclude that minimal idempotents of abundant holomorphic semigroup $S$ are just those in $K(S)$, that is, they are from $E(S) \cap K(S)$. Theorem 2.3 is also an important source of alternative definition of minimal idempotent. That is, on the basis of Theorem 2.3 (2), minimal idempotent can also be defined as follows. An idempotent e on semigroup $S$ is minimal if it belongs to the minimal left (or right) ideal. Also, from the same Theorem 2.3 (3), we can say that minimal left (or right) ideal always is of the form $S \circ e$ (or $e \circ S$ ), where $e$ is a minimal idempotent in $S$.

\section{References}

[1] Bergelson, V., 2003, Minimal idempotents and ergodic Ramsey theory, Ergodic Theory of $\mathbb{Z}^{d}$ action, Topics in dynamics and ergodic theory: London Math. Soc. Lecture Note Ser. 310, 8-39.

[2] Hinkkanen, A., \& Martin, G. J., 1996, The dynamics of semigroups of rational functions-I. Proc. London Math. Soc., 73 (3): 358-384.

[3] Poon, K. K., 1998, Fatou-Julia theory on transcendental semigroups. Bull. Austral. Math. Soc., 58: 403-410.

[4] Subedi, B. H., \& Singh, A., 2018, Escaping sets of hyperbolic semigroups. The Nep. Math. Sci. Report, 35 (1 \& 2): 45-52. arXiv:1803.10381 v1 [math DS].

[5] Subedi, B. H., \& Singh, A., 2019, Fatou, Julia, and escaping sets in holomorphic (sub)semigroup dynamics. Turk. J. Math., 43 (2): 930-940. DOI: 10. 3906/mat-1810-133. arXiv: 1807.04499v1 [math. DS].

[6] Subedi, B. H., \& Singh, A., 2019, Fatou, Julia, and escaping sets of conjugate holomorphic semigroups. The Nep. Math. Sci. Report, 36 (1-2): 61-66. arXiv:1808.03275v1 [math DS]. 